\title{
SOME RECENT DEVELOPMENTS OF REGENERATIVE CIRCUITS*
}

\author{
$\mathrm{BY}$ \\ Edwin H. Armstrong \\ (Marcellus Hartley Research Laboratory, Columbia University, \\ New York)
}

It is the purpose of this paper to describe a method of amplification which is based fundamentally on regeneration, but which involves the application of a principle and the attainment of a result which it is believed is new. This new result is obtained by the extension of regeneration into a field which lies beyond that hitherto considered its theoretical limit, and the process of amplification is therefore termed super-regeneration.

Before proceeding with a description of this method it is in order to consider a few fundamental facts about regenerative circuits. It is well known that the effect of regeneration (that is, the supplying of energy to a circuit to reinforce the oscillations existing therein) is equivalent to introducing a negative resistance reaction in the circuit, which neutralizes positive resistance reaction, and thereby reduces the effective resistance of the circuit. There are three conceivable relations between the negative and positive resistances: namely-the negative resistance introduced may be less than the positive resistance, it may be equal to the positive resistance, or it may be greater than the positive resistance of the circuit.

We will consider what occurs in a regenerative circuit containing inductance and capacity when an alternating electromotive force of the resonant frequency is suddenly impressed for each of the three cases. In the first case (when the negative resistance is less than the positive), the free and forced oscillations have a maximum amplitude equal to the impressed electomotive force over the effective resistance, and the free oscillation has a damping determined by this effective resistance. The steady state is attained after the initial free oscillation dies out and continues until the impressed emf. is removed, when the current dies out

*Received by the Editor, June 3,1922. Presented before ThE Institute of Radio Engineers, New York, June 7, 1922. 
in accordance with a second free oscillation. The maximum amplitude of current in this case is always finite; it reaches this maximum amplitude in a finite time, and when the impressed emf. is removed the current dies away to zero. This is the action of the circuits which are now in every-day practical use.

In the second case the negative resistance is equal to the positive resistance, and the resultant effective resistance of the circuit is therefore zero. When an emf. is suddenly impressed in this case, the current in the circuit starts to increase at a rate which is directly proportional to the impressed electromotive force and to the square root of the ratio of the capacity to the inductance of the circuit (for a given impressed frequency). If the force is impressed for an infinite time, then the current in the eircuit reaches infinity. If the emf. is impressed for a finite time, then the current reaches some finite value. When the impressed emf. is removed, the current in the circuit at that instant continues indefinitely with unchanged amplitude as a free oscillation. Theoretically, this is the limiting case for regeneration; practically, it is always necessary to operate at some point slightly below this state at which the circuits have a definite resistance.

It is important to note here that altho the circuit of this case has zero resistance, oscillations will not start unless an emf. is impressed upon the circuit; furthermore, that oscillations once started continue with undiminished amplitude indefinitely. This state cannot be attained in practice, because the negative resistance furnished by the tube is dependent on the amplitude of the current and for stable operation decreases with increasing amplitude. ${ }^{1}$

In the third case the negative resistance introduced into the circuit is greater than the positive resistance, and the effective resistance of the circuit is therefore negative. When an emf. is impressed upon a circuit in this condition, a free and a forced oscillation are set up which have some interesting properties. The amplitude of the forced oscillation is determined by the value of the impressed emf. divided by the resultant resistance of the circuit. The free oscillation starts with an amplitude equal to the forced oscillation, and builds up to infinity regardless of whether

${ }^{1}$ It is very important at this point to distinguish between this purely theoretical state and the state which exists in oscillating tube circuits. In the various forms of self-heterodyne circuits a free oscillation of constant amplitude is maintained in the system and the circuit may be considered as having zero resistance, but only for that particular amplitude of current. An external emf. impressed on the circuit always encounters a positive resultant resistance, assuming, of course, that the existing oscillation is stable. This is due to the non-linear characteristic of the tube. 
or not the external emf. is removed. This free oscillation starts with an amplitude which is proportional to the impressed force; and this proportionality is maintained thruout any finite time interval (with constant impressed electromotive force).

It is important to note that altho the negative resistance of the circuit exceeds the positive, and the effective resistance of the circuit is negative, oscillations will not occur until some emf. is impressed. Once an emf. is impressed, however, no matter how small it may be, the current in the circuit builds up to infinity regardless of whether or not the external emf. is removed.

The fundamental difference between the case in which the resistance of the circuit is positive and the case in which the resistance of the circuit is negative may be summed up as follows: in the first; the forced oscillation contains the greatest amount of energy and the free oscillation is of very minor importance ${ }^{2}$ (after a short interval of time), in the second, it is the free oscillation which contains the greatest amount of energy and the forced oscillation which is of negligible importance.

It is, of course, impossible with present-day instrumentalities to set up a system in which the negative resistance exceeds the positive without the production of oscillations in the system, since any irregularity in filament emission or impulse produced by atmospheric disturbances is sufficient to initiate an oscillation which builds up to the carrying capacity of the tube. It is, however, possible, by means of various expedients, to set up systems which avoid the production of such a paralyzing oscillation and which approximate the theoretical case in the use of a free oscillation to produce amplification.

The first use of the free oscillation in a regenerative system for the amplification of signals appears to have been made by Turner $^{3}$ in his valve relay system. Briefly, Turner prevented the regenerative circuit from producing oscillations when no signals were being received by placing a negative potential on the grid of sufficient value to hold it just below that point on the characteristic curve at which self-oscillation would start. The impressing of a small electromotive force of sufficient value would carry the potential of the grid over the "threshhold" value and a free oscillation would start which would build up to the limiting

${ }^{2}$ This is strictly true when dealing with continuous waves which we have been considering. It is not true in the regenerative reception of spark signals, particularly of short wave length, large damping, and low spark frequency. In this case the energy in the free oscillation exceeds the energy in the forced oscillation.

'British Patent, 130,408. 
value of the tube. The system was returned to its initial sensitive state by means of a relay operated by the increase in the plate current of the tube. This relay short-circuited the feedback coil, thereby cutting off the supply of energy and permitting the potential of the grid to drop back below the "threshhold" value. As Turner explains, the device is a relay with a low limit (as distinguished from an amplifier), but it appears to be the first device in which the free oscillation set up by an impressed electromotive force produced the magnified result.

Bolitho ${ }^{4}$ contributed an important improvement by replacing the mechanical relay of Turner which operated only upon the receipt of a signal by a valve relay which was continuously operated by independent means. Briefly, this was accomplished by connecting a second valve to the oscillating circuit of the Turner arrangement with a reversed feed-back connection and supplying the plate circuit of this second valve with alternating current. When the "threshhold" value of the first tube was overcome and a free oscillation started in the system, the reversed feed-back of the second tube comes into action and at that time when the voltage supplied to the plate is positive, damps out the free oscillation and permits the grid of the first tube to return below the "threshhold" value. This represents the second step in the utilization of the free oscillation for the production of amplification.

It is the purpose of this paper to describe a principle of operation based on the free oscillation which is quantitative and without a lower limit. This new method is based on the discovery that if a periodic variation be introduced in the relation between the negative and positive resistance of a circuit containing inductance and capacity, in such manner that the negative resistance is alternately greater and less than the positive resistance, but that the average value of resistance is positive, than the circuit will not of itself produce oscillations, but during those intervals when the negative resistance is greater than the positive will produce great amplification of an impressed emf. The free oscillations which are set up during the periods of negative resistance are directly proportional in amplitude to the amplitude of the impressed emf. The variation in the relation between the negative and positive resistance may be carried out by varying the negative resistance with respect to the positive, by varying the positive resistance with respect to the negative, or by varying both simultaneously at some frequency which is generally rela-

${ }^{4}$ British Patent, 156,330. 
tively low compared to the frequency of the current to be amplified.

These three methods of producing the super-regenerative state are illustrated respectively by Figures 1,2 , and 3 , which figures indicate the general scheme of the system and the methods of varying the relation between the negative and positive resistance. Figure 1 shows a method of varying the negative resistance produced by the regenerative system by varying the voltage of the plate of the amplifying tube by means of a second tube, the grid of the second tube being excited by an emf. of suitable frequency.

Figure 2 illustrates a method of varying the positive resistance of the circuit with respect to the negative. This is accomplished by connecting the plate circuit of a vacuum tube in parallel to the tuned circuit of the regenerative system and exciting the grid by an emf. of suitable frequency. Figure 3 illustrates a combination of these two systems in which simultaneous varia-

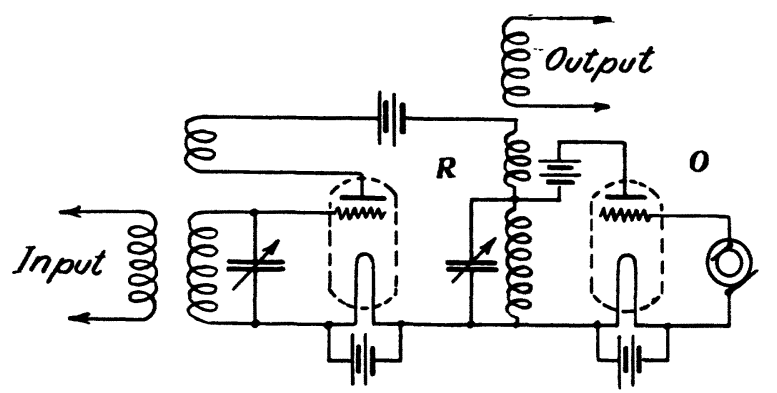

Figure 1

tions are produced in both the negative and positive resistances and provision made for adjusting the relative phases of these two variations.

A general idea of the phenomena occurring in these systems when an emf. is applied to the input circuit will be obtained from the diagram of Figure 4 which applies specifically to the circuit of Figure 1. This figure illustrates the principle relations existing in the system in which the positive resistance is constant and the variation is introduced into the negative resistance. It will be observed that the frequency of variation appears as a modulation of the amplified current so that the output circuit contains currents of the impressed frequency plus two side frequencies differing from the fundamental by the frequency of the variation.

Oscillograms of the essential current and voltage relations existing in the systems of the type illustrated by Figures 1 and 2 


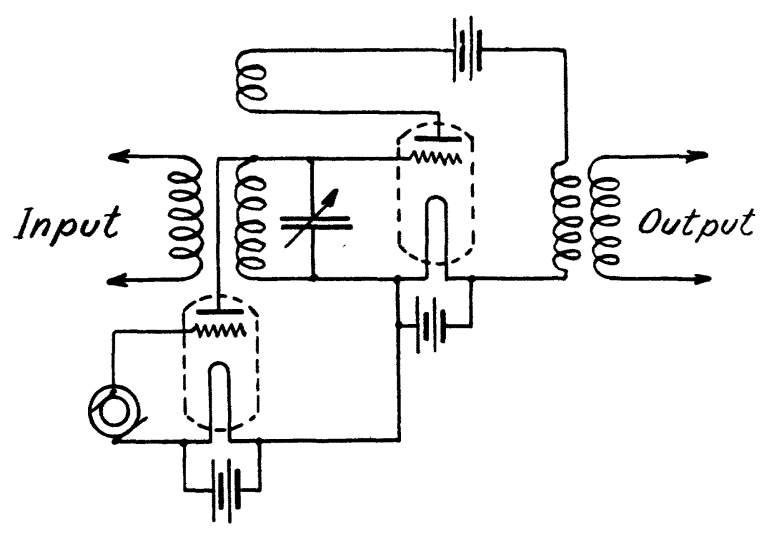

Figure 2

were obtained with the set up of apparatus illustrated in Figures 5 and 6 , respectively. In the arrangement of Figure 6 , in order to produce sufficient variation in the positive resistance of the tuned circuit, which was of large capacity and low inductance, it

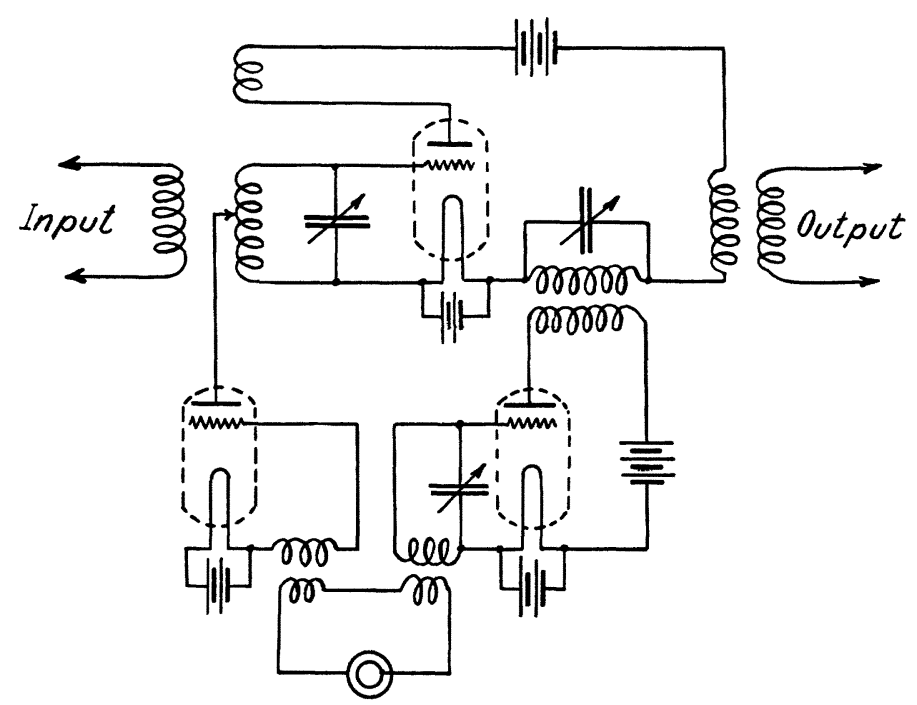

Figure 3

was necessary to use a two-electrode tube in series with the auxiliary emf.

Figures 7 and 8 are oscillograms respectively for a negative resistance variation and a positive resistance variation. The signaling emf. was impressed about half way along the film, the 


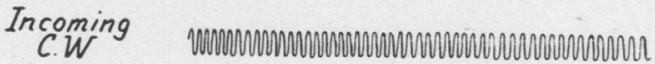
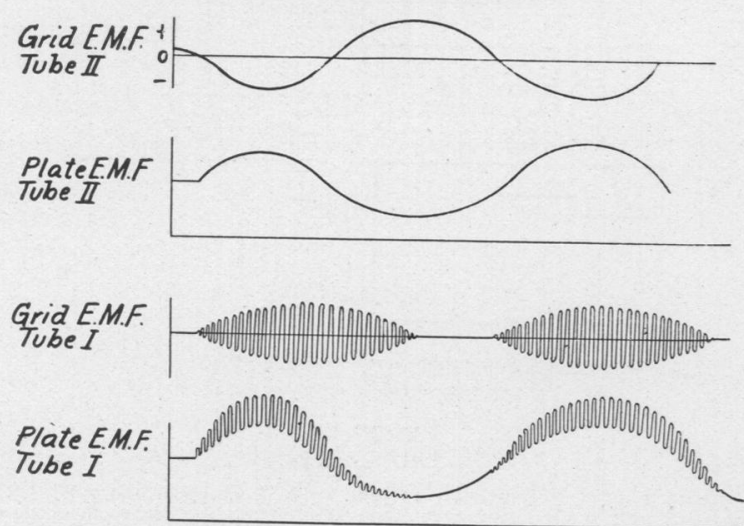

Output

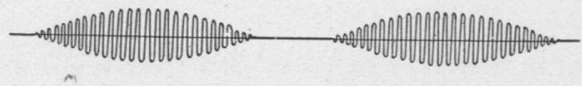

\section{Rectified \\ Current}

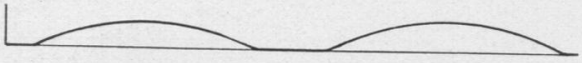

Figure 4

Tube $\mathrm{I}$ refers to $\mathrm{R}$ in Figure 1

Tube II refers to $\mathrm{O}$ in Figure 1

exact point at which the key was closed being indicated by the arrow. These oscillograms show phenomena which are in accordance with the explanations already given, but, in addition, show evidence of self excitation. It has been stated in the preceding pages of this paper that the basis of super-regeneration was the discovery that a variation in the relation between the

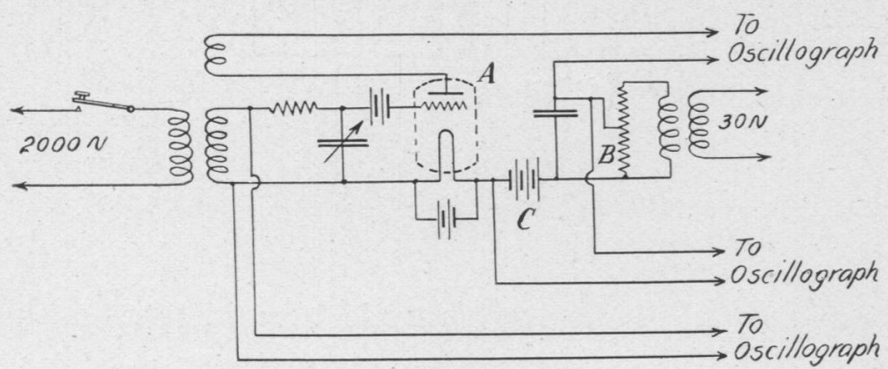

Figure 5

A-4 Western Electric Type L Tubes in parallel

B-AC Voltage $=100$ Volts

C-DC Voltage $=160$ Volts 


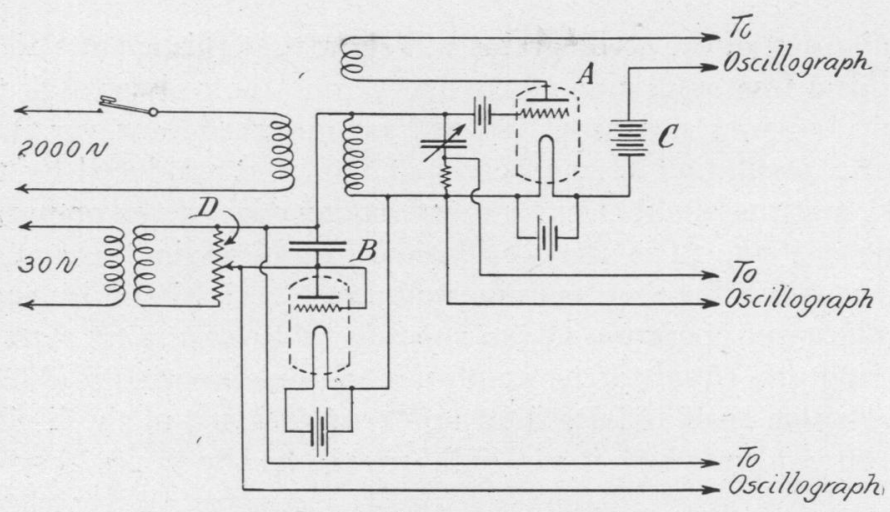

Figure 6

A-4 Western Electric Type L Tubes in parallel

B-1 Western Electric Type L Tubes with grid and plate in parallel C-DC Voltage $=160$ Volts

$\mathrm{D}-\mathrm{AC}$ Voltage $=30$ Volts

negative and positive, resistances prevented a system which would normally oscillate violently from becoming self-exciting. An

Grid Circuit

Current

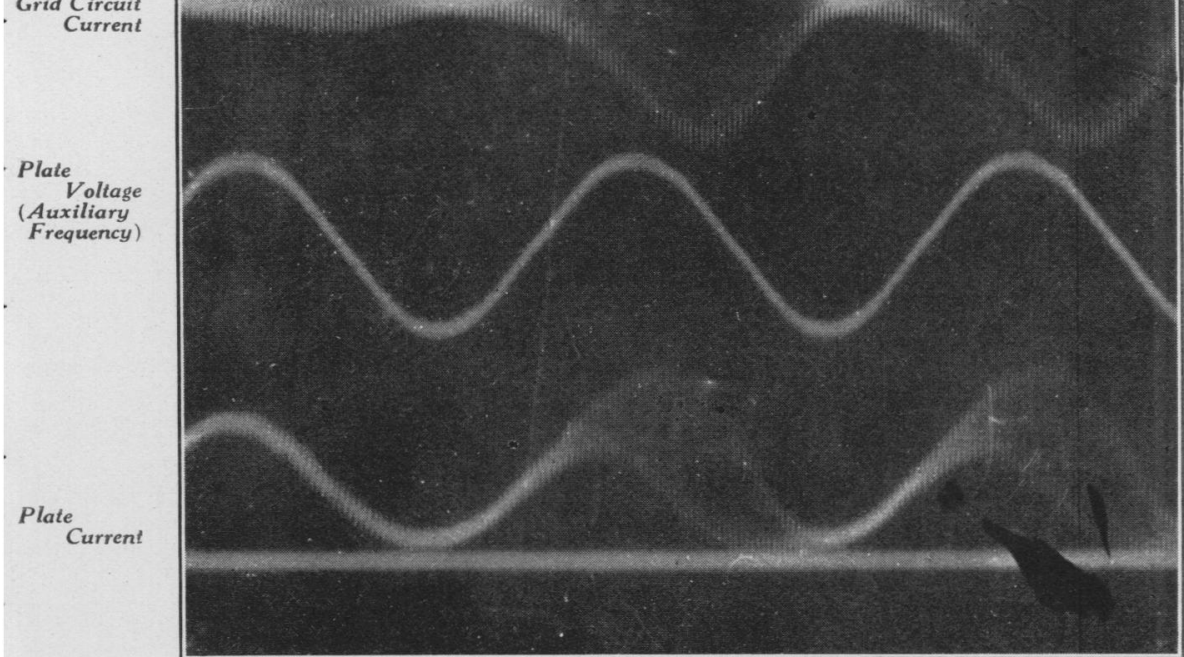

Figure 7 


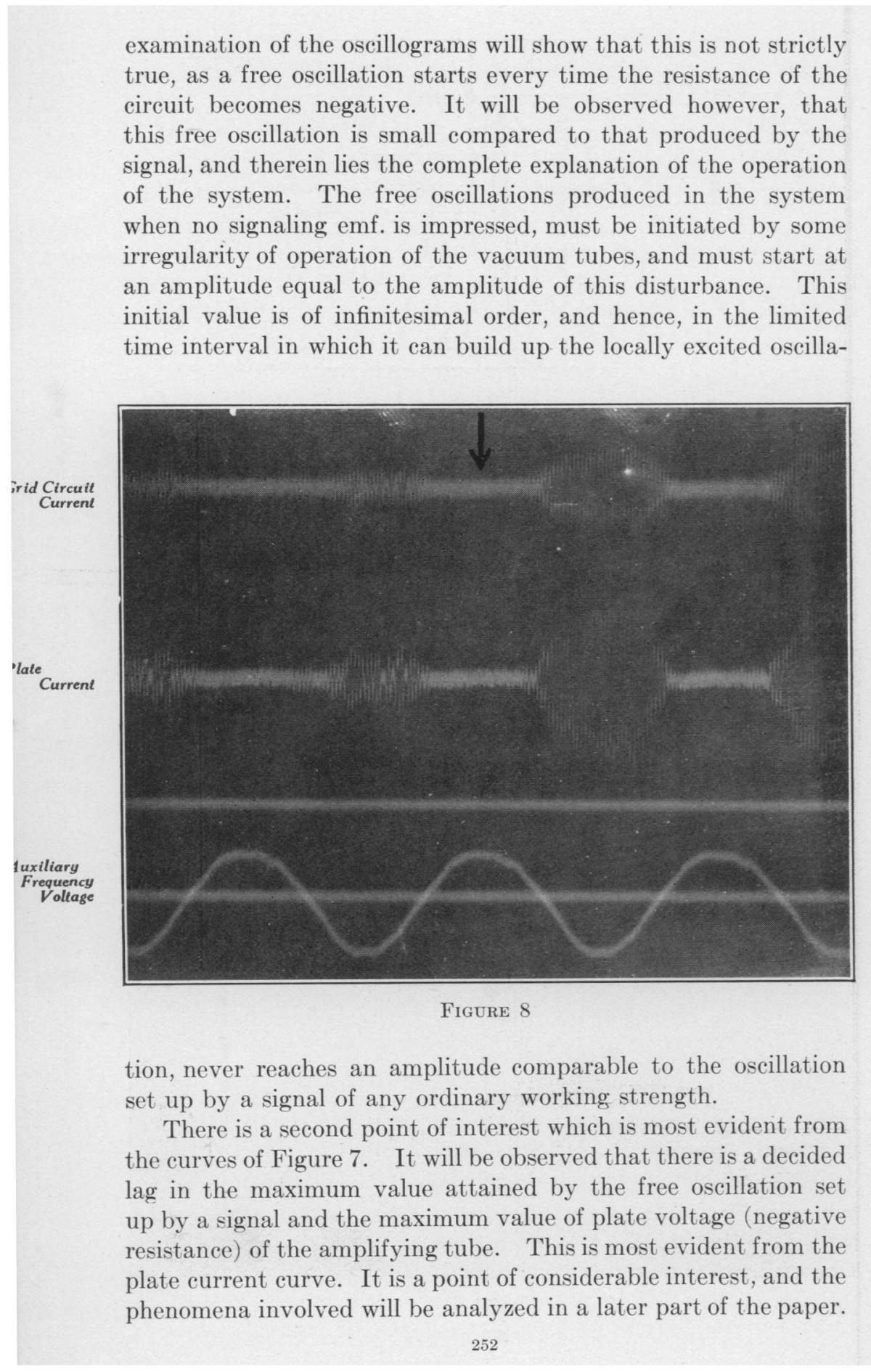


The rate of variation in the relation between the negative and positive resistance is a matter of great importance. It may be at sub-audible, audible, or super-audible frequencies. In radio signaling, for the reception of telephony, the variation should be at a super-audible frequency. For modulated continuous wave telegraphy and spark telegraphy, to retain the tone characteristics of the signals, it must be well above audibility; for maximum amplification a lower and audible rate of variation should be used. In continuous wave telegraphy, where an audible tone is required, the variation is at an audible rate; where the operation of an indicating device is required, a sub-audible frequency may be best. The choice of frequency is a compromise, particularly in telephony, since obviously the lower the frequency the greater the amplification, and the higher the frequency the better the quality.

Some practical forms of circuits are illustrated by Figures 9,10 , and 11, which illustrate respectively the three types of variation. Figure 9 shows a method of varying the plate voltage of the amplifying tube $R$ by means of the vacuum tube oscillator $O$ coupled into the plate circuit. In this arrangement a third tube $D$ acts as a detector. This is essential when an audible frequency is employed; when a super-audible frequency is used the telephones can be placed directly in the plate circuit of the amplifying tube.

Figure 10 shows the second case in which the variation is

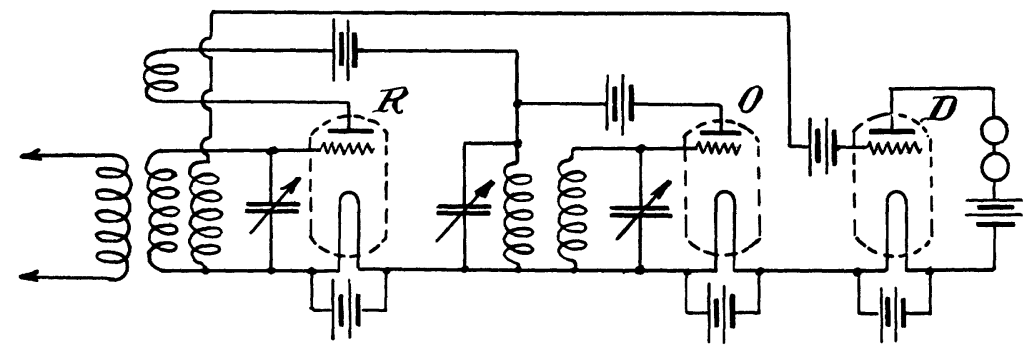

Figure 9

introduced into the positive resistance of the tuned circuit. This is done by means of an oscillating tube $O$, the grid circuit of which is connected thru the tuned circuit $L C$ of the amplifying tube $R$. The variation in the resistance of the circuit is effected thru the variation in potential of the grid of the oscillating tube. During that half of the cycle, when the grid of the oscillating ube is positive, energy is withdrawn from the tuned circuit in 
the form of a conduction current from the grid to the filament of the oseillating tube, thereby increasing the effective resistance of the circuit. During the other half of the cycle, when the grid of the oscillating tube is negative, no conduction current can flow thru the grid circuit of the oscillating tube, and hence no resistance is introduced into the tuned circuit of the amplifying tube. In this case the amplifying tube serves also as the detector for any frequency of variation, as the tuned circuit forms a sufficiently good filter even for an audible frequency to prevent a disturbing audible tone in the telephones.

Figure 11 illustrates the case of a simultaneous variation in both positive and negative resistances. This is accomplished by providing the amplifying tube $R$ with a second feed-back circuit $L_{1} C_{1}$ and $L_{2} C_{2}$ adjusted to oscillate at some lower frequency, thereby introducing a variation in the negative resistance thru the variation of the plate potential of the amplifier and a varia-

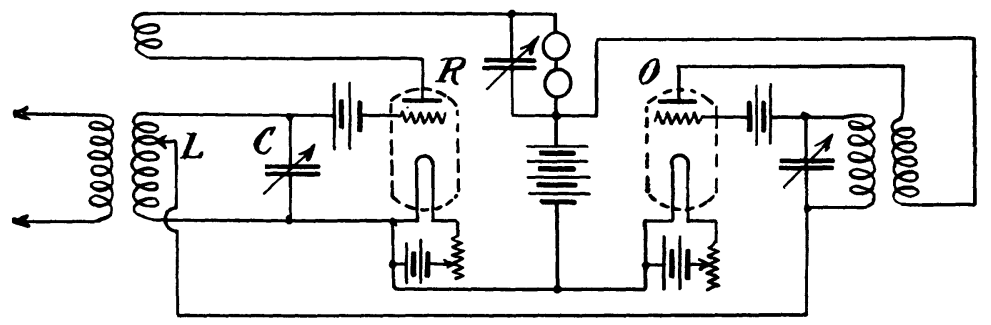

Figure 10

tion in the positive resistance by means of the variation of the grid of the amplifier. The proper phase relations between the negative and positive resistance are obtained by adjustment of the capacity of condensers $C_{1}$ and $C_{2}$ and the coupling between $L_{1}$ and $L_{2}$. In operation this system is very critical, and extreme care is necessary in order to obtain the super-regenerative state.

In each of the preceding cases the detecting function has been carried out either by a separate tube or by means of the amplifying tube. When a super-audible frequency of variation is employed, it is sometimes of advantage to perform the detecting function in the oscillating tube, and an arrangement for carrying this out is illustrated in Figure 12. The operation of this system is as follows: incoming signals are amplified by means of the regenerative action of the amplifier tube $R$ and the variations of potential across the tuned wave frequency circuit $L C$ impressed upon the grid of the oscillating tube $O$. These oscillations are 


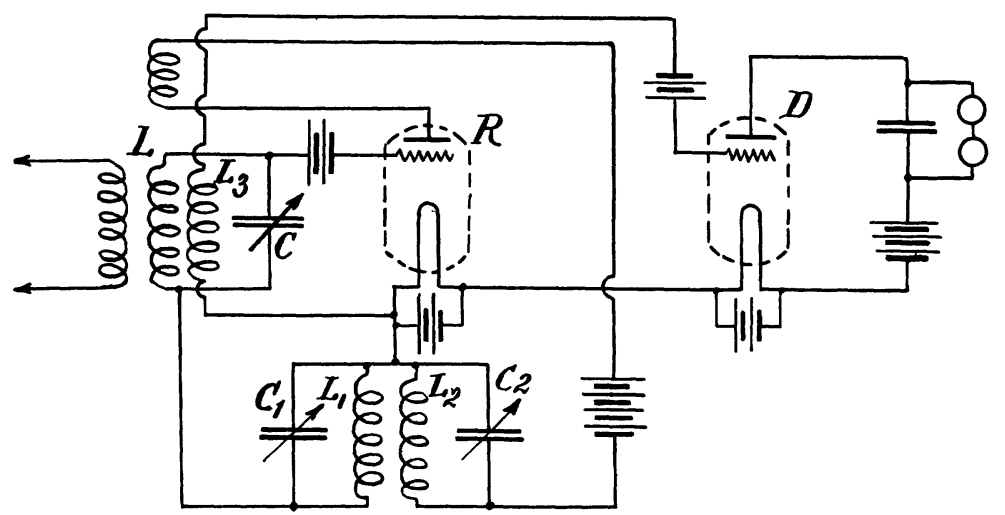

Figure 11

then rectified, and two frequencies are produced in the circuits of the amplifier tube. One of these frequencies corresponds to the frequency of modulation of the signaling wave. The other corresponds to the frequency of the variation and contains a modulation in amplitude corresponding to the modulation of the transmitted wave. This second frequency is then impressed upon the circuits of the oscillating tube with which it is in tune, amplified by the regenerative action of the system $L_{1} C_{1} L_{2} O$, and then rectified. The amplification obtainable with this form of

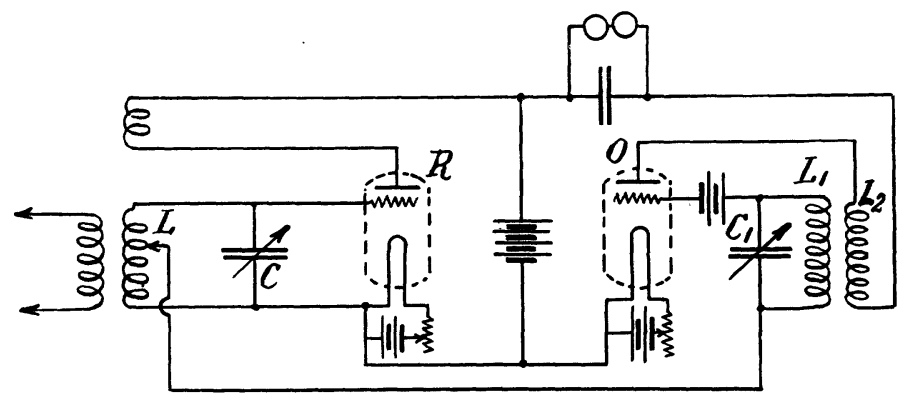

Figure 12

system is considerably greater than that of the single amplification circuits, but is naturally more complicated to operate.

When a super-audible variation is employed in a system such as illustrated in Figure 1, it is generally necessary to introduce a certain amount of resistance in the tuned circuit to insure the dying out of the free oscillation during the interval when the resistance of the circuit is positive. This is most effectively. carried out by means of the arrangement illustrated in Figure 
13 , in which a secondary coil $L_{1}$ of large inductance and high resistance is coupled to the tuned circuit $L C$ and the energy withdrawn thereby from the oscillating circuit stepped up and applied to the grid of the tube. In the operation of this system, a curious phenomena is encountered. This is the manifestation of an inductive reaction by the plate circuit of the amplifying tube to the auxiliary frequency emf. supplied the plate circuit

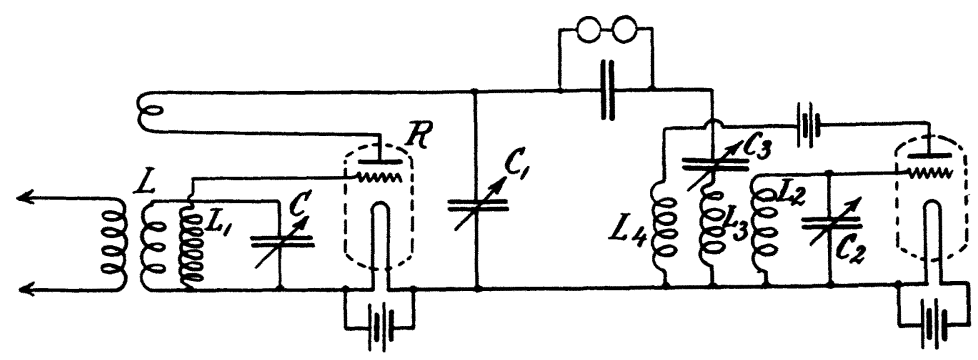

FIGURE 13

by the oscillating tube, which comes about in the following way When the auxiliary emf. is impressed upon the plate of the amplifying tube, a current is produced in this tube in phase with the emf. across the tube. Now suppose the plate voltage is at its maximum positive value. This means that the negative resistance of the circuit is a maximum in amplitude. This in turn means that the average value of the grid is becoming more positive and the current in the plate circuit is likewise increasing. Since the free oscillation in the system will increase in amplitude as long as the resistance of the circuit is negative, it will reach its maximum amplitude after the maximum positive voltage is applied to the plate. Hence the component of current corresponding to the frequency of the variation set up in the plate circuit by the rectification of the radio frequency oscillations lags in phase behind the auxiliary emf. impressed on the plate. Hence the plate circuit of the tube manifests an inductive reaction to the auxiliary emf. It was found that this inductive reaction could be tuned out by means of the parallel condenser $C_{1}$ with a great improvement in the stability of the operation of the system and increase in the signal strength. The resonance point is pronounced, and once the other adjustments of the system have been correctly made is as readily found as any ordinary tuning adjustment.

The problem of cascade amplification with these systems is a rather involved one on account of a great number of effects which 
are not encountered in ordinary methods of cascade amplification. The principal trouble is the reaction of the second amplifying system on the first, and the difficulty of preventing it in any simple way on account of the high amplification per stage. While this difficulty is not insuperable, a simple expedient may be employed which avoids it. On account of the large values of radio frequency energy in these amplifying systems, the second harmonic is very strong in the plate circuit of the amplifying tube and is of the same order of magnitude as the fundamental if the tube is operated with a large negative voltage on the grid. Hence by arranging the second stage of a cascade system to operate at double the frequency and to amplify this harmonic, the difficulty is avoided. The general arrangement of such a system is illustrated by Figure 14, in which the positive resistance of the eireuits $L C$ and $L_{1} C_{1}$ of a two-stage amplifier are varied synchronously by a single oscillator. The circuit $L_{1} C_{1}$ in this case is tuned to the second harmonic of the circuit $L C$, but the combinations of circuits which may be arranged on this principle are very numerous.

One of the curious phenomena encountered with the superregenerative system is found when it is attempted to secure

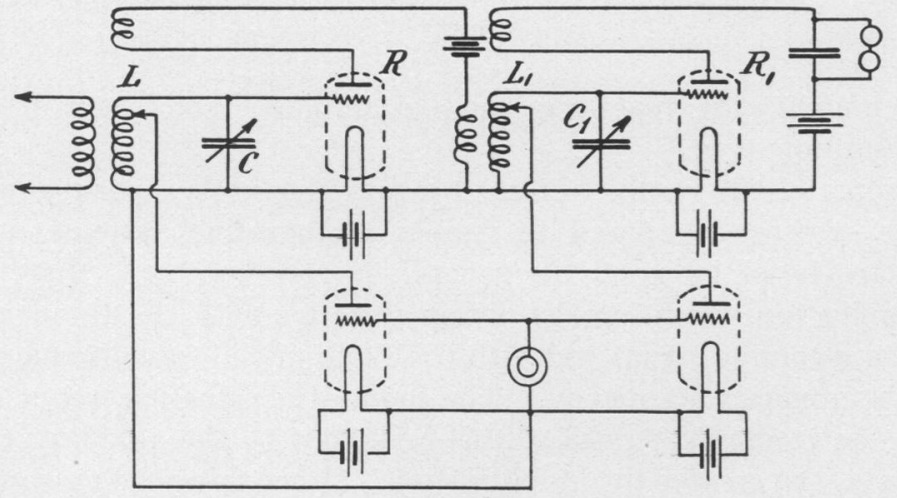

Figure 14

sharp tuning by the use of tuned circuits placed between the antenna and the amplifying system. The free oscillations set up in these circuits by the reaction of the amplifying system continue in these circuits during the interval when the resistance of the amplifier circuit is positive, re-excite the amplifier when the resistance becomes negative, and hence the entire system is kept in a continuous state of oscillation. The effect is most critical, 
and may be produced with most extremely weak couplings between the amplifier circuit and the second tuned circuit. The simplest solution of the difficulty is to perform the function of tuning at one frequency and amplification at another, and this is best accomplished by means of the super-heterodyne method illustrated by Figure 15. This may be adapted to work on either the sum or difference frequencies, but when the higher frequency is used, care should be taken that it is not near the second harmonic of the local heterodyning current. In the particular arrangement illustrated, $L C D$ represents, together with the heterodyne, the usual agency for changing the incoming frequency, and

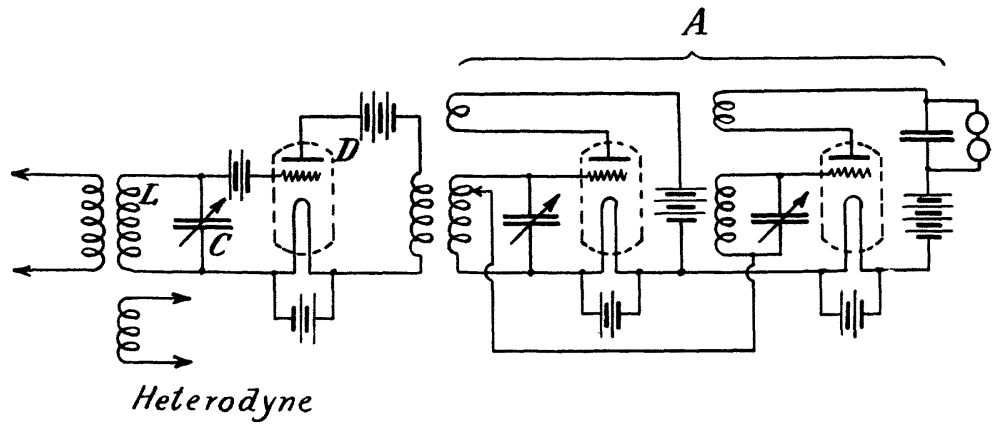

Figure 15

$A$ represents the super-regenerative amplifier which may be of any suitable type.

Some of the results obtained in practice with super-regenerative systems compared to simple regenerative systems may perhaps be of interest. In general, it may be stated that the amplification which can be obtained varies with the frequency of the incoming signal and with the radio of the wave frequency to the auxiliary frequency. The higher the signaling frequency and the greater the ratio of this frequency to the auxiliary frequency, the greater the amplification. Other things being equal, it appears that the energy amplification varies as the square of the ratio of the signaling frequency to the auxiliary frequency. Hence, it follows that for telegraphic signals where an audible auxiliary frequency is used, much greater amplification can be obtained than in the case of telephony, where a super-audible auxiliary frequency must be employed. Using the arrangement of Figure 11 for a signaling frequency' of five hundred thousand cycles, an energy amplification several million times greater than that obtainable with a simple self-heterodyne circuit is 
readily secured. Where a super-audible frequency is used for the reception of telephone signals, amplification of fifty thousand to one hundred thousand times energy can be obtained.

In a practical way the relative amplification of the new system with respect to the standard regenerative system for reception of telephone signals may be visualized as follows: With a signal so extremely weak that only the faintest of beat notes can be heard in the ordinary regenerative receiver, the super-regenerative receiver will give clearly understandable speech. For signals of sufficient strength to be understandable with the ordinary regenerative system with zero beat adjustment but not audible without local oscillations, the superregenerative receiver will produce signals loud enough to be heard thruout the room.

Perhaps the most surprising characteristic of the system, apart from the amplification, is its selectivity with respect to spark interference when a super-audible frequency of variation is used. The explanation of this selectivity with respect; for example, to the ordinary regenerative receiver, lies in the periodic suppression of all free vibrations in the system. In the ordinary regenerative system spark interference approximates a form of shock excitation setting up a free vibration in the system which, because of the low damping existing therein, continues for a long period of time. An examination of the character of the oscillation set up will show that the energy existing in the free vibration after the initial impressed electromotive force is removed, is far greater than the forced vibration. In the ordinary system this free vibration may exist for a thousandth of a second or more. In the super-regenerative system this free vibration is damped out before it has proceeded more than one twenty thousandth of a second as a maximum. Hence, the interference from spark signals is greatly reduced. This phenomenon opens up a new field for the suppression of interference produced by shock excitation.

At the present time, on a three-foot loop antenna located twenty-five miles from the station WJZ at Newark, New Jersey, and a system of the type illustrated in Figure 12 with one stage of audio frequency amplification (three tubes in all) the announcements and musical selections are clearly audible five hundred yards from the receiver. With the same loop at the same distance, using the arrangement of Figure 11 without the separate detector tube, that is, with the telephones directly in the plate circuit of the amplifier tube, it is possible to operate a loud- 
speaking telephone so that the program from the Newark station is plainly heard thru a large size room. The signals with the arrangements of either Figures 11 or 12 are still heard loudly if the loop is discontinued from the receiver, the coils and wires of the receiver itself collecting sufficient energy to produce response.

W'hile the new system does not amplify the ordinary spark signal with anything approaching its efficiency on continuous wave signals, one example of spark reception may be of interest. During the past winter an amateur spark station located at Cleveland, Ohio, and operating on a wave length of about 340 meters was received nightly at Yonkers, New York, on a threefoot (1 meter) loop and the arrangement of Figure 13 with sufficient intensity to enable the signals to be read thruout the room.

In conclusion, I wish to express my very great indebtedness to Professor L. A. Hazeltine for much valuable aid in connection with the theoretical side and to Mr. W. T. Russell for his assistance thruout the experimental side of this development.

\section{June 2, 1922. Marcellus Hartley Research Laboratory, Columbia University, New York.}

SUMMARY: A system of circuits is described whereby the effective resistance of a regenerative circuit is periodically made positive and negative, tho predominantly positive. Such a circuit will respond to impressed electromotive forces by setting up free oscillations during the negative resistance period, which oscillations are proportional to the exciting emf. The forced oscillations produced by the exciting emf. will be comparatively small. The free oscillations caused by any normal variations in tube operation will also be small.

Means of carrying regenerative circuits thru the requisite resistance cycles are shown, and the practical operation of the system, including the case when extreme amplifications are desired, is discussed. 